\title{
Anisotropic Homogeneous Cosmologies in the Post-Newtonian Approximation.
}

\author{
Tamath Rainsford \\ Department of Physics and Mathematical Physics, \\ University of Adelaide, South Australia 5005, Australia
}

\begin{abstract}
In this paper we explore how far the post-Newtonian theory, [9] goes in overcoming the difficulties associated with anisotropic homogeneous cosmologies in the Newtonian approximation. It will be shown that, unlike in the Newtonian case, the cosmological equations of the post-Newtonian approximation are much more in the spirit of general relativity with regard to the nine Bianchi types and issues of singularities.

The situations of vanishing rotation and vanishing shear are treated separately. The homogeneous Bianchi I model is considered as an example of a rotation-free cosmology with anisotropy. It is found in the Newtonian approximation that there are arbitrary functions that need to be given for all time if the initial value problem is to be well-posed, while in the post-Newtonian case there is no such need. For the general case of a perfect fluid only the post-Newtonian theory can satisfactorily describe the effects of pressure. This is in accordance with findings in [7] where the post-Newtonian approximation was applied to homogeneous cosmologies.

For a shear-free anisotropic homogeneous cosmology the Newtonian theory of Heckmann and Schücking, [2] is explored. Comparisons with its relativistic and post-Newtonian counterparts are made. In the Newtonian theory solutions exist to which there are no analogues in general relativity. The post-Newtonian approximation may provide a way out.
\end{abstract}




\section{Introduction}

Due to its simplicity, the Newtonian approximation of cosmology is preferable to general relativity and is typically used wherever possible. However, in a recent paper [9], it is shown that the Newtonian approximation has some difficulties. To begin with the Newtonian approximation is incomplete in the sense that the Bianchi identities cannot be obtained from the field equations. It is generally accepted that Newtonian theory is a specialization of the linearized approximation of general relativity cut off at the $c^{-2}$-level. However, information is lost in this cut-off procedure, and it is shown that cutting off at the $c^{-4}$-level results in a theory in which the Bianchi identities are obtainable from the field equations, 9]. Moreover, the Newtonian theory has no initial value problem when applied to cosmology because there are no boundary conditions. Therefore, the Poisson equation has no unique solution. The post-Newtonian theory, on the other hand, is a closed, consistent and well-posed theory. The post-Newtonian approximation used here is obtained by expanding up to order $c^{-6}$ and reformulating the linearized field equations as wavelike equations. A specialization of the resulting equations leads to a formally well-posed $c^{-4}$-theory. This $c^{-4}$-approximation is the first order approximation to give us consistency and formal well-posedness and, as we shall see, suffices as a "Newtonian" approximation to cosmology. Going to higher order simply gives increasingly more accurate approximations of general relativity.

In [7] it was shown that the post-Newtonian approximation, in the context of the $k=0$ Friedmann-Robertson-Walker cosmology (FRW), is able to fully reproduce the results of its general relativistic counterpart, whilst the Newtonian theory is not. The Newtonian approximation of cosmology does not allow the pressure to enter into the dynamics. Hence, changing the equation of state, does not result in different solutions for the density and expansion. In fact, the only solution that agrees with general relativity is that for the matter dominated universe. On the other hand, in the post-Newtonian approximation pressure becomes dynamic through an extra function of time, $A(t)$. Hence, the post-Newtonian theory is able to produce differing solutions for alternative equations of state in accordance with general relativity. In the case where $A(t)$ goes to zero the pressure is forced to vanish and the higher order terms of the post-Newtonian approximation vanish as well, giving exactly the same solution for dust as the Newtonian theory does. Thus, Newtonian theory for homogeneous and isotropic cosmology should only be used for the special case of dust, where the two theories, the Newtonian and post-Newtonian, coincide.

In this paper we explore how far the post-Newtonian approximation goes in overcoming the difficulties of the Newtonian theory when applied to the more general case of anisotropic homogeneous cosmology. There is a great deal of theoretical and observational evidence to support anisotropy in the universe, [5]. Any theory which attempts to approximate general relativity should yield similar results to the fully relativistic theory. The FRW models are isotropic and homogeneous. Although they fit most of the observed features of the universe they do not fit all. Different geometries may be worth considering at earlier times, for example, near the initial singularity. There might exist a general rotation of matter in the universe of comparable magnitude to the general expansion that we can not detect. Shear might provide a deviation away from isotropy since extra-galactic objects might be observed by the galactic equator [3]. All homogeneous cosmologies fall into two classes: the Bianchi models, which are those for which the isometry group admits a 3-dimensional simply transitive subgroup, and those for which the isometry group is neither simply transitive, nor admits a simply transitive subgroup - the Kantowski-Sachs models. There exist nine Bianchi types and, correspondingly, nine Bianchi cosmologies, each class has subclasses with extra symmetries. The Bianchi types are in general anisotropic, so they do not have all spatial directions at a point being equivalent. Instead, there are now accelerations, and anisotropic observers will no longer be orthogonal to the 
surfaces of homogeneity. Not all Bianchi types are suitable for describing the universe. They were first considered as cosmological models by Taub, [11] then shortly followed by Raychaudhuri, 8] and Heckmann and Schücking, [4. These models contain the FRW models as a special case. Thus, they are considered the simplest and the most likely cosmological candidates, in particular Bianchi I, Bianchi IX and the LRS types $7_{o}$ and $7_{h}$.

Bianchi I has a different cosmic scale factor for each direction and only becomes isotropic in the case where the scale factors all become equal. In this case the Bianchi I model becomes the $k=0$ FRW cosmology. For nonzero shear these rotation-free anisotropic models are called the Heckmann-Schücking solutions, [2].1 In the vacuum case they become the Kasner solution.

Unfortunately, there are solutions in the case of anisotropy to which there are no general relativistic analogues. Theorems of Ellis, [1] tell us that shear-free perfect fluids have either vanishing expansion or vanishing rotation. The case of vanishing rotation just yields the FRW model which is explored in [7]. The case of vanishing expansion, however, is more problematic since in Newtonian cosmology it allows for singularity-free solutions. This in in contradiction with theorems of Hawking which state that solutions of general relativity must have singularities. The post-Newtonian approximation provides a way out of this difficulty.

We begin in section 2.1 with a review of homogeneous Newtonian cosmology. Then in 2.2 we consider post-Newtonian approximation to homogeneous anisotropic cosmology. We find that the Newtonian theory is in general under-determined and unable to fully reproduce the nine Bianchi types of general relativity. But the post-Newtonian equations produce a set of cosmological equations which are more in the spirit of the Bianchi identities.

In section 3 we consider the case where the rotation vanishes separately. Bianchi I is taken as an example of a rotation-free anisotropic and homogeneous cosmology, and explored in the Newtonian and post-Newtonian contexts. In section 4 we consider the shear-free case. We end in section 5 with a conclusion.

\footnotetext{
${ }^{1}$ Not to be confused with the Newtonian Heckmann-Schücking cosmologies.
} 


\section{Newtonian Theory and the Post-Newtonian Approximation in Homogeneous Anisotropic Cosmologies}

\subsection{Newtonian Homogeneous Anisotropic Cosmology}

In the following we will consider the Newtonian cosmological model of Heckmann and Schücking, [2]. For a homogeneous cosmology the density, $\rho$, and the pressure, $p$, are functions of time only. The velocity field, $v_{i}$, is the same relative to all observers and such that $v_{i}=V_{i j}(t) X_{j},[6$, , 2, 10]. The theory is then described by the Poisson, continuity and Euler equations, which are given by:

$$
\begin{aligned}
\phi_{, i i} & =4 \pi G \rho, \\
\dot{\rho}+\rho v_{i, i} & =0 \\
\dot{v}_{i}+\phi_{, i} & =0
\end{aligned}
$$

where $G$ is the Newtonian gravitational constant. Here $\bullet$ refers to the convective derivative, $\dot{\bullet}=\frac{\partial}{\partial t} \bullet+v_{i} \bullet, i$

We find from (3) that the Newtonian potential $\phi$, up to a constant, takes the form $\phi=$ $a_{i j}(t) X_{i} X_{j}$. Thus equation (1) simplifies to

$$
a_{i i}=4 \pi G \rho
$$

We make the following decomposition

$$
V_{i j}=\frac{1}{3} \theta \delta_{i j}+\sigma_{i j}+\omega_{i j}
$$

with

$$
\begin{aligned}
\theta & =V_{i i}, \\
\sigma_{i j} & =\frac{1}{2}\left(V_{i j}+V_{j i}\right)-\frac{1}{3} \theta \delta_{i j}, \\
\omega_{i j} & =\epsilon_{j i k} \omega_{k}=\frac{1}{2}\left(V_{i j}-V_{j i}\right),
\end{aligned}
$$

where the trace, $\theta$ is the expansion, the trace-free symmetric part $\sigma_{i j}$ is the shear and the antisymmetric part $\omega_{i j}$ is the rotation. Inserting these expressions for $V_{i j}$, equations (2) and (3) become

$$
\dot{\rho}+\rho \theta=0,
$$

and

$$
\begin{aligned}
\dot{\theta} & =4 \pi G \rho-\frac{1}{3} \theta^{2}+2 \omega^{2}-2 \sigma^{2}, \\
\dot{\omega}_{i} & =-\frac{2}{3} \theta \omega_{i}+\sigma_{i j} \omega_{j}, \\
\dot{\sigma}_{i j} & =-\frac{2}{3} \theta \sigma_{i j}-\sigma_{i k} \sigma_{k j}-\omega_{i k} \omega_{k j}+\frac{2}{3}\left(\sigma^{2}-\omega^{2}\right) \delta_{i j}-a_{i j}+\frac{1}{3} a_{k k} \delta_{i j},
\end{aligned}
$$

where $\sigma^{2}=\frac{1}{2} \sigma_{i j} \sigma_{i j}$ and $\omega^{2}=\frac{1}{2} \omega_{i j} \omega_{i j}=\omega_{i} \omega_{i}$.

Equations (4) and (6)-(9), along with a barotropic equation of state relating the density and pressure, form the Newtonian approximation to cosmology. The structure of these equations is as follows: There are eleven equations, one each arising from the Poisson, continuity and the 
trace of the Euler equation, five from the symmetric trace-free part and a further three from the antisymmetric part. However, there are 16 unknowns: $\theta, \rho, \omega_{i}, \sigma_{i j}$ and $a_{i j}$. Thus, the system is under-determined and it is not possible to solve uniquely for all the unknowns.

Providing five arbitrary functions of time, for instance by specifying the shear $\sigma_{i j}(\mathrm{t})$, for all time, we are able to solve for the five functions $\theta, \rho$ and $\omega$, the Newtonian potential $\phi=$ $a_{i j}(t) X_{i} X_{j}$ and the pressure $p$ through the barotropic equation of state. Then, for a set of initial data $\left(\omega_{i}\left(x_{i}, 0\right), \rho\left(t_{0}\right), \phi\left(t_{0}\right)\right)$ the set of homogeneous Newtonian cosmologies may be found since (6), (4) and (8) can be uniquely solved for $\theta, \omega_{i j}$ and $\rho$, and, $a_{i i}$ can be determined from (44) and $a_{i j}$ from (9).

This is very different to the fully general relativistic case where there are nine possible symmetry groups. In general relativity the system of equations which describe cosmology has a well-posed Cauchy problem. In the Newtonian case, however, the system is under-determined - at least five functions of the set $\theta, \omega_{i}$ and $\sigma_{i j}$ must be specified for all time. Thus, the Newtonian theory is not able to fully reproduce the full Bianchi types of general relativity in general. Arbitrary shear has no analogy in general relativity. However, once five (arbitrary) constraints have been imposed, the theory has an initial value formulation within the constraint of being homogeneous and isotropic and within the strange set up of allowing a given evolution of the shear.

Let us explore how far the post-Newtonian approximation [9] goes in overcoming this difficulty. The post-Newtonian theory is known to be well-posed in the general case of anisotropy and inhomogeneity [9]. The extra potentials of the post-Newtonian theory act as integrability conditions for the Newtonian potential $\phi$. We will see how these extra potentials help us in making the system determined in general.

\subsection{Post-Newtonian Approximations of Anisotropic Homogeneous Cosmolo- gies}

Following a scheme similar to that of Weinberg [12], we adopt units in which the typical velocity has magnitude 1 , i.e. $\beta \approx \frac{v}{c}$, and assume a one parameter family of metrics $g_{\mu \nu}\left(x^{\lambda}, c\right)$ for which there is a system of coordinates $\left(x^{0}, x^{i}\right)$ in which the components of the metric have the following asymptotic behaviour as $c \longrightarrow \infty$ :

$$
\begin{aligned}
g_{00} & =-1-2 \phi c^{-2}-2 \alpha c^{-4}-2 \alpha^{\prime} c^{-6}-2 \alpha^{\prime \prime} c^{-8} \ldots \ldots \\
g_{0 i} & =\zeta_{i} c^{-3}+\zeta_{i}^{\prime} c^{-5}+\zeta_{i}^{\prime \prime} c^{-7} \ldots . . \\
g_{i j} & =\delta_{i j}-2 \phi \delta_{i j} c^{-2}+\alpha_{i j} c^{-4}+\alpha_{i j}^{\prime} c^{-6}+\alpha_{i j}^{\prime \prime} c^{-8} \ldots \ldots
\end{aligned}
$$

The usual Newtonian theory is obtained as the $\mathcal{O}\left(c^{-2}\right)$ limit of $(10)$, while the Newtonian approximation is the $\mathcal{O}\left(c^{-4}\right)$ limit. Reformulating the field equations of the $\mathcal{O}\left(c^{-6}\right)$ limit as wavelike equations defines the post-Newtonian theory used here (see [9]).

After applying the harmonic gauge conditions

$$
\begin{aligned}
\dot{\phi} & =-\frac{1}{4} \zeta_{i, i}, \\
\dot{\zeta}_{i} & =\phi_{i j, j},
\end{aligned}
$$

the field equations for the post-Newtonian theory are

$$
\begin{aligned}
\phi_{, k k} & =4 \pi G \rho+\frac{1}{4 c^{2}}\left(-\phi_{j k, j k}-J\right), \\
\zeta_{i, k k} & =16 \pi G \rho v_{i}+\frac{1}{c^{2}}\left(\dot{\phi}_{i j, j}-K_{i}\right), \\
\ddot{\phi}_{i j}-c^{2} \phi_{i j, k k} & =K_{i j}+c^{2}\left[16 \pi G\left(\rho v_{i} v_{j}+\delta_{i j} p\right)-J_{i j}\right],
\end{aligned}
$$


where the $\alpha$ and $\alpha_{i j}$ of (10) are such that

$$
\phi_{i j}=\alpha_{i j}-2 \delta_{i j} \alpha
$$

with

$$
\begin{aligned}
J \equiv & 6 \phi_{, i} \phi_{, i}-16 \pi G\left(\rho v^{2}+4 \rho \phi\right) \\
K_{i} \equiv \quad & 3 \zeta_{j, j} \phi_{, i}+2 \zeta_{j} \phi_{, i j}-2 \phi_{, j} \zeta_{j, i} \\
& -16 \pi G\left[v_{i} p+\rho v_{i} v^{2}-\frac{1}{2} \rho \zeta_{i}\right] \\
J_{i j} \equiv & 8 \phi \phi_{, i j}+4 \phi_{, i} \phi_{, j}-\delta_{i j}\left(6 \phi_{, k} \phi_{, k}+32 \pi G \rho \phi\right), \\
K_{i j} \equiv & -\frac{1}{2}\left(\zeta_{i} \zeta_{k, k j}+\zeta_{j} \zeta_{k, k i}\right)-\zeta_{k}\left(\zeta_{i, j k}+\zeta_{j, i k}\right)+2 \zeta_{k} \zeta_{k, i j}+\zeta_{k, i} \zeta_{k, j}+\zeta_{i, k} \zeta_{j, k} \\
& -2 \phi_{, k}\left(\phi_{k i, j}+\phi_{k j, i}-2 \phi_{i j, k}\right)-16 \phi \phi_{, i} \phi_{, j}+\phi_{, i} \phi_{k k, j} \\
& +\phi_{, j} \phi_{k k, i}-2 \phi\left(\phi_{k i, j k}+\phi_{k j, i k}-\phi_{i j, k k}-\phi_{k k, i j}\right)-2 \phi_{k i} \phi_{, j k} \\
& -2 \phi_{k j} \phi_{, i k}+2 \phi_{, i j} \phi_{k k}-\delta_{i j}\left(\frac{1}{2} \zeta_{k, m} \zeta_{k, m}+\frac{1}{2} \zeta_{m, k} \zeta_{k, m}+\frac{1}{2}\left(\zeta_{k, k}\right)^{2}\right. \\
& -\zeta_{k} \zeta_{m, m k}-4 \phi_{, k} \phi_{k m, m}+4 \phi_{, k} \phi_{m m, k}-12 \phi \phi_{, k} \phi_{, k} \\
& \left.-\phi\left(2 \phi_{k m, m k}-2 \phi_{m m, k k}\right)\right) \\
& +8 \pi G\left[2 p v_{i} v_{j}+2 \rho\left(2 \phi+v^{2}\right) v_{i} v_{j}+\rho \phi_{i j}\right. \\
& \left.+\delta_{i j}\left(2 \rho \phi v^{2}-\frac{1}{2} \phi p+\frac{3}{4} \phi_{, k} \rho_{, k}+\frac{1}{2} \rho \phi_{, k k}\right)\right]
\end{aligned}
$$

This system forms a closed set which is consistent because the Bianchi identities are obtainable from the field equations:

$$
\begin{aligned}
& \dot{\rho}\left(1+\frac{v^{2}-4 \phi}{c^{2}}\right)+\left(\rho v_{j}\right)_{, j}\left(1+\frac{v^{2}}{c^{2}}\right)+\frac{1}{c^{2}}\left[\rho\left(2 v_{j} \dot{v}_{j}+2 v_{j} v_{k} v_{k, j}+\frac{1}{2} \zeta_{j, j}\right)\right. \\
& \left.-\frac{1}{2} \rho_{, j} \zeta_{j}+\left(v_{j} P\right)_{, j}+\frac{1}{16 \pi G}\left(2 \phi_{, i} \zeta_{i, j j}-2 \zeta_{i} \phi_{, j i i}-3 \zeta_{i, i} \phi_{, j j}\right)\right]=0
\end{aligned}
$$

and

$$
\begin{aligned}
& \rho\left(\dot{v}_{i}+v_{i, j} v_{j}+\phi_{, i}\right)+P_{, i}=\frac{1}{16 \pi G c^{2}}\left[-\left(\dot{J}+K_{j, j}\right) v_{i}+\right. \\
& \left.\dot{K}_{i}-K_{i j, j}-2 \phi\left(J_{, i}+\phi_{j k, k i j}\right)-\phi_{, i}\left(J+\phi_{j k, j k}\right)\right] .
\end{aligned}
$$

Homogeneity can be provided by the following ansätze:

$$
\begin{aligned}
\phi & =a_{i j}(t) X_{i j}+a(t), \\
\zeta_{i} & =b_{i j k l}(t) X_{j k l}+b_{i j}(t) X_{j}, \\
\phi_{i j} & =c_{i j k l m n}(t) X_{k l m n}+c_{i j k l}(t) X_{k l}+c_{i j}(t),
\end{aligned}
$$

where $X_{i j}=X_{i} X_{j}, X_{i j k}=X_{i} X_{j} X_{k}$ etc. These are more general ansätze than in [7] where isotropy has been ensured through similar constraints. As before we assume that the density and pressure are functions of time only, and are related through a barotropic equation of state. The velocity field decomposes as shown before in equation (5). 
Substituting the ansätze (18) into the field equations for the post-Newtonian theory, Eqs. (13 to 15), and comparing expressions of different powers in $X_{i}$, yields the set of equations for the anisotropic homogeneous post-Newtonian approximation:

$$
\begin{aligned}
& a_{k k}=2 \pi G \rho+c^{-2}\left[-\frac{1}{4} c_{k l k l}+8 \pi G \rho a\right], \\
& 3\left(2 a_{i k} a_{j k}+c_{k l k l i j}\right)-4 \pi G \rho\left(4 a_{i j}+V_{k i} V_{k j}\right)=0,
\end{aligned}
$$

$$
3 b_{i k k j}=8 \pi G \rho V_{i j}+c^{-2}\left[\dot{c}_{i k k j}-3 b_{k k} a_{i j}-2 a_{i k} b_{k j}+2 a_{j k} b_{k i}+8 \pi G\left(V_{i j} p-\frac{1}{2} \rho b_{i j}\right)\right],
$$

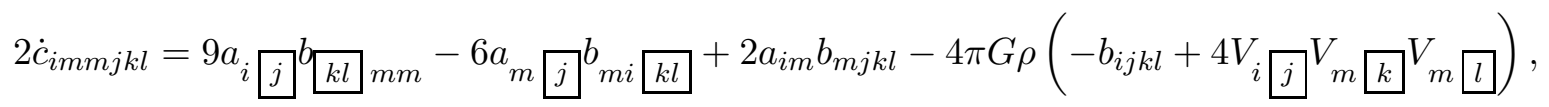

$$
\begin{aligned}
& 2 c_{i j k k}-16 a a_{i j}+16 \pi G(2 \rho a+p) \delta_{i j}+c^{-2}\left[-\ddot{c}_{i j}+2 b_{i k} b_{j k}+4 a_{i j} c_{k k}-4 a_{i k} c_{j k}-4 a_{j k} c_{i k}\right. \\
& \left.-\delta_{i j}\left(\frac{1}{2} b_{k k} b_{l l}+b_{k l} b_{k l}\right)+8 \pi G\left(\rho c_{i j}+\delta_{i j}\left(-\frac{1}{2} a p+\frac{1}{2} \rho c_{k k}\right)\right)\right]=0,
\end{aligned}
$$

$$
\begin{aligned}
& 12 c_{i j m n k k}-8\left(2 a_{i j} a_{m n}+a_{i m} a_{j n}+a_{i n} a_{j m}-3 \delta_{i j} a_{m k} a_{n k}\right)+32 \pi G \rho \delta_{i j} a_{m n} \\
& +8 \pi G \rho\left(V_{i m} V_{j n}+V_{i n} V_{j m}\right) \\
& +c^{-2}\left[-\ddot{c}_{i j m n}+16 a\left(3 \delta_{i j} a_{k m} a_{k n}-2 a_{i m} a_{j n}-2 a_{i n} a_{j m}\right)\right. \\
& +\frac{3}{2}\left(2 b_{i n} b_{k k j m}+2 b_{j n} b_{k k i m}+2 b_{i m} b_{k k j n}+2 b_{j m} b_{k k i n}-b_{i k} b_{j k m n}-b_{j k} b_{i k m n}\right) \\
& +2\left(a_{i n} c_{k k j m}+a_{j n} c_{k k i m}+a_{i m} c_{k k j n}+a_{j m} c_{k k i n}\right)-4\left(a_{i k} c_{k j m n}+a_{j k} c_{i k m n}-a_{i j} c_{k k m n}\right) \\
& +4 \pi G\left(2 \rho c_{i j m n}+p V_{i m} V_{n j}+p V_{i n} V_{m j}+p V_{j m} V_{n i}+p V_{j n} V_{m i}\right. \\
& \left.\left.\quad+4 \rho a\left(V_{i m} V_{j n}+V_{j m} V_{i n}+\delta_{i j} V_{l m} V_{l n}\right)-p a \delta_{i j} a_{m n}+\delta_{i j} c_{m n k k}\right)\right]=0,
\end{aligned}
$$

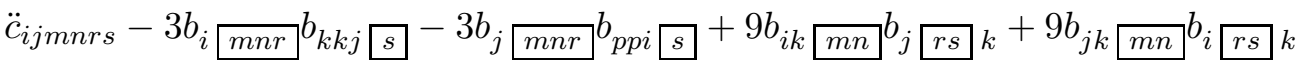

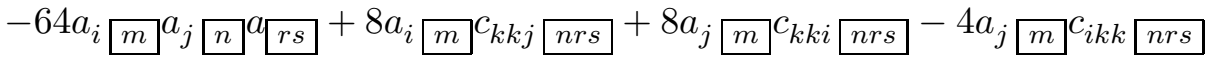

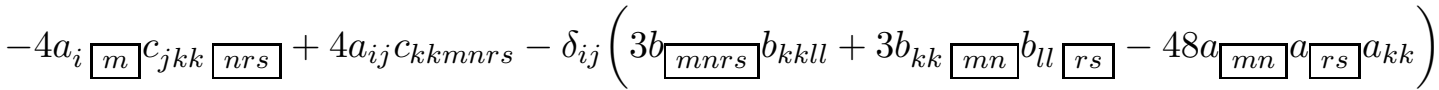

$$
\begin{aligned}
& +8 \pi G\left[4 \rho a_{[m n} V_{i r r} V_{j ! s}+\rho c_{i j m n r s}+2 \rho V_{i m} V_{j n} V_{[r s}\right.
\end{aligned}
$$

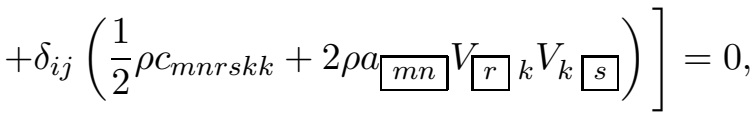


where we have introduced the notation

$$
\begin{aligned}
b_{i\lfloor m n} b_{p p j \leq s}=\frac{1}{12} & \left\{b_{i m n r} b_{p p j s}+b_{i m r s} b_{p p j n}+b_{i m n s} b_{p p j r}+b_{i n m r} b_{p p j s}\right. \\
& +b_{i n r s} b_{p p j m}+b_{i n m s} b_{p p j r}+b_{i r m n} b_{p p j s}+b_{i r m s} b_{p p j n} \\
& \left.+b_{i r n s} b_{p p j m}+b_{i s m n} b_{p p j r}+b_{i s m r} b_{p p j n}+b_{i s n r} b_{p p j m}\right\}
\end{aligned}
$$

and similar, to indicate total symmetrisation in all indices that are surrounded by a box.

The harmonic gauge conditions (11, 12) give

$$
\begin{aligned}
\dot{a} & =-\frac{1}{4} b_{k k}, \\
\dot{a}_{i j} & =-\frac{3}{4} b_{k k i j}, \\
\dot{b}_{i j} & =2 c_{i k k j}, \\
\dot{b}_{i j k l} & =4 c_{i m m j k l},
\end{aligned}
$$

and the Bianchi identities become

$$
L(t)+c^{-2}\left(M(t)+N_{i j}(t) X_{i j}\right)=0
$$

and

$$
O_{i j} X_{j}+c^{-2}\left(Q_{i j}(t) X_{j}+S_{i j k l}(t) X_{j k l}\right)=0,
$$

where

$$
\begin{aligned}
& L(t)=\dot{\rho}+\rho V_{k k}, \\
& M(t)=-4 \dot{\rho} a+\frac{1}{2} \rho b_{k k}+p V_{k k}-\frac{3}{8 \pi G} a_{k k} b_{l l}, \\
& N_{i j}(t)=\rho\left(V_{i k} V_{j k} V_{l l}+V_{i k} \dot{V}_{j k}+V_{j k} \dot{V}_{i k}+V_{i k} V_{k l} V_{l j}+V_{j k} V_{k l} V_{l i}+\frac{3}{2} b_{k k i j}\right) \\
& +\frac{1}{2} \dot{\rho}\left(V_{i k} V_{k j}+V_{j k} V_{k i}-8 a_{i j}\right)+\frac{1}{16 \pi G}\left[\frac{7}{2} a_{i k} b_{k l l j}+\frac{7}{2} a_{j k} b_{k l l i}-18 a_{k k} b_{l l i j}\right] . \\
& O_{i j}(t)=\dot{V}_{i j}+V_{i k} V_{k j}+2 a_{i j} \\
& Q_{i j}(t)=\frac{1}{\rho}\left\{V_{i j}\left(4 \dot{\rho} a+4 \rho \dot{a}-\frac{1}{2} \rho b_{k k}-\rho a V_{k k}-\dot{p}\right)+24 \rho a a_{i j}-p \dot{V}_{i j}+\frac{1}{2} \dot{\rho} b_{i j}+\frac{1}{2} \rho \dot{b}_{i j}\right. \\
& \left.-(p+\rho a) V_{i k} V_{k j}+\frac{p}{2} a_{i j}+2 \rho a V_{k i} V_{k j}-\rho c_{i k k j}\right\} \\
& +\frac{1}{16 \pi G \rho}\left\{-6 V_{i j} a_{k k} b_{l l}+6 \dot{b}_{k k} a_{i j}+6 b_{k k} \dot{a}_{i j}+4 a_{i k} \dot{b}_{k j}+4 \dot{a}_{i k} b_{k j}-96 a a_{i k} a_{j k}\right. \\
& -48 a c_{k l k l i j}+3 b_{i j} b_{k k l l}-3 b_{i k} b_{l l k j}-6 b_{i k} b_{k l l j}+6 b_{k j} b_{i k l l}-6 b_{k i} b_{l l k j} \\
& +9 b_{k k} b_{l l i j}-9 b_{k j} b_{l l i k}-6 b_{k l} b_{k l i j}+6 b_{k l} b_{l k i j}-12 a_{i j} c_{k l k l}+8 a_{i j} c_{k k l l}-8 a_{i k} c_{k l l j} \\
& \left.+4 a_{i k} c_{l l k j}+4 a_{j k} c_{l l k i}+8 a_{k l} c_{k l i j}-8 a_{k j} c_{i l k l}-4 a_{k k} c_{l l i j}\right\}
\end{aligned}
$$

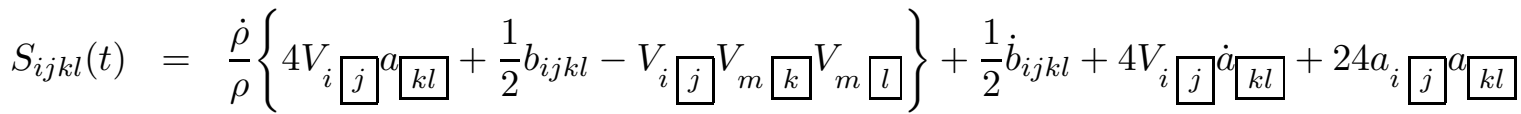




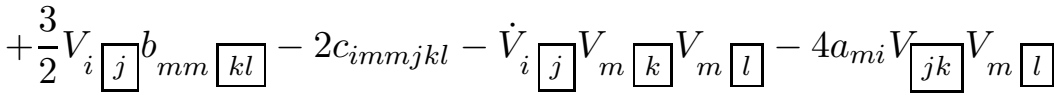

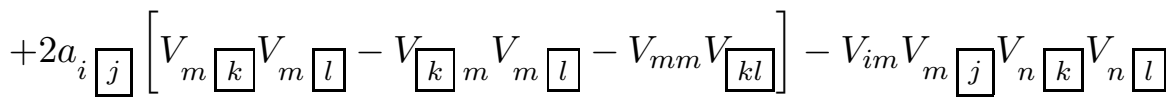

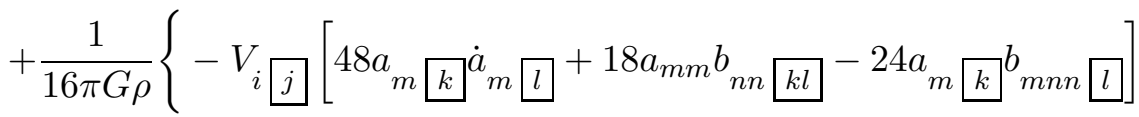

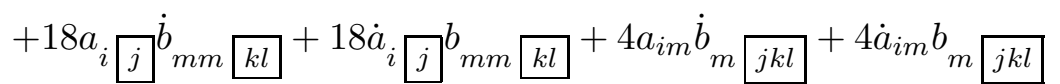

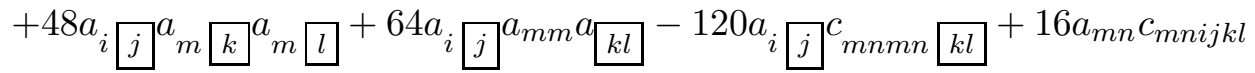

$$
\begin{aligned}
& -48 a_{i m} c_{j n n} k l-8 a_{m m} c_{n n i j k l}+56 a_{i m} c_{n n m j k l}-16 a_{i m} c_{m m n j k l} \\
& +24 a_{i[j}{ }_{m m n n}{ }_{k l}-9 b_{i j k m} b_{n n m !}+3 b_{i j k l} b_{m m n n}+6 b_{i m n n} b_{m j k l} \\
& -18 b_{m n i} b^{b}{ }_{m n}{ }_{k l}-18 b_{m n n i} b_{m j k l}-27 b_{m m n i} b_{n j k l}
\end{aligned}
$$

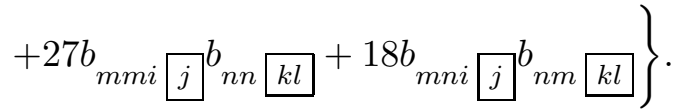

Note that setting $L(t)=0$, i.e. setting higher order terms in $c^{-2}$ to zero, one recovers the continuity equation of the Newtonian approximation. Similarly, $O(t)=0$ yields the Euler equation of the Newtonian approximation.

It can be shown that the time derivative of (19) and the trace of equation (21) combined give

$$
L(t)+c^{-2} M(t)=0,
$$

in accordance with the Bianchi identity (16). This essentially is the continuity equation with $c^{-2}$ corrections. The time derivative of equation (20) and the trace of equation (22) yield

$$
N_{i j}(t) X_{i j}=0
$$

which is also consistent with the Bianchi identity (16).

We can also recover the post-Newtonian Euler equation by combining the time derivative of (21) and the diagonal elements of (24) to obtain

$$
O_{i j}(t) X_{j}+c^{-2} Q_{i j}(t) X_{j}=0
$$

The final part

$$
S_{i j k l} X_{j k l}(t)=0,
$$

can be obtained by combining the time derivative of (22) with equation (25).

Thus we may completely define the anisotropic, inhomogeneous post-Newtonian cosmology with the set of equations; (29), (31), (19), (20), (21), (22), (25), (24, $m \neq n$ ) and (23). 2 In the case of the isotropic, homogeneous post-Newtonian cosmology, [7] it was shown that there existed relationships between the field equations rendering many of them redundant. This is special to the case of isotropy and due to the symmetries of the potentials. We know very little about the symmetries of the potentials in the more general situation of anisotropy. The harmonic gauge conditions provide a little assistance. For example:

Newtonian cosmology is contained within the equations (29), (31), (19), (20), (21), (22), (25), (24, $m \neq n$ ) and (23), as the special case when the $c^{-2}$ terms go to zero. Equations (19), (29) and

\footnotetext{
${ }^{2}$ Eq. (23) is the only equation not to contribute to the Bianchi identities.
} 
(31) are then the Newtonian theory with $c^{-2}$ corrections. The corrections to the theory contain extra unknowns - the potentials $\zeta_{i}=b_{i j k l} X_{j k l}+b_{i j} X_{j}$ and $\phi_{i j}=c_{i j k l m n} X_{k l m n}+c_{i j k l} X_{k l}+c_{i j}$ which are determined by the equations (20), (21), (22), (25), (24, $m \neq n)$ and (23).

We will now attempt to use the equations (29), (31), (19), (20), (21), (22), (25), (24, $m \neq n$ ) and (23), to solve for the unknowns; $a_{i j}(t), a(t), b_{i j k l}(t), b_{i j}(t), c_{i j k l m n}(t), c_{i j k l}(t)$ and $c_{i j}(t)$.

Let us start with equation (21) which has trace

$$
3 b_{l k k l}=8 \pi G \rho V_{k k}+c^{-2}\left(\dot{c}_{l k k l}-3 a_{k k} b_{l l}+8 \pi G\left[p V_{k k}-\frac{1}{2} \rho b_{k k}\right]\right) .
$$

With the aid of the harmonic gauge conditions (26), it can be shown that this is just the time derivative of equation (19). Thus this equation is redundant. The symmetric part of (21) is

$$
\begin{aligned}
& \frac{3}{2}\left(b_{i k k j}+b_{j k k i}\right)+\frac{4}{3} \dot{a}_{k k} \delta_{i j}=8 \pi G \rho \sigma_{i j} \\
& \left.\quad+c^{-2}\left\{\frac{1}{4}\left[\ddot{b}_{i j}+\ddot{b}_{j i}\right]+\frac{2}{3} \dddot{a} \delta_{i j}+12 \dot{a} a_{i j}-4 \dot{a} a_{k k} \delta_{i j}+2 \pi G\left[4 p \sigma_{i j}-\rho\left(b_{i j}+b_{j i}\right)-\frac{8}{3} \rho \dot{a} \delta_{i j}\right)\right]\right\},
\end{aligned}
$$

where the harmonic gauge conditions and equation (19) have been used. The antisymmetric part is given by

$$
\begin{aligned}
& 8 \pi G \rho \omega_{i j}+\frac{3}{2}\left(b_{j k k i}-b_{i k k j}\right) \\
& \quad+c^{-2}\left\{\frac{1}{4}\left[\ddot{b}_{i j}-\ddot{b}_{j i}\right]-2 a_{i k} b_{k j}+2 a_{j k} b_{k i}+2 \pi G\left[4 p \omega_{i j}-\rho\left(b_{i j}-b_{j i}\right)\right]\right\}=0,
\end{aligned}
$$

where once again the harmonic gauge conditions have been used for simplification where ever possible. These equations can be solved once we know $a_{i j}$ to obtain information about $b_{i j}{ }^{[}$and $b_{i j k l}$.

We now want to find $a_{i j}$. To do so we consider equations (20), (22) and (25). Equation (20) has the trace

$$
\ddot{a}_{k k}=6 a_{k l} a_{k l}-4 \pi G \rho\left\{4 a_{k k}+\frac{1}{3} \theta^{2}+2 \sigma^{2}+2 \omega^{2}\right\}
$$

providing us with an equation for $a_{k k}$. The traceless part is given by

$$
6 a_{i k} a_{j k}-\ddot{a}_{i j}-4 \pi G \rho\left(4 a_{i j}+\frac{1}{9} \theta^{2}+2 \sigma^{2}+2 \omega^{2}+\frac{2}{3} \theta \sigma_{i j}+\sigma_{k i} \omega_{k j}+\sigma_{k j} \omega_{k i}\right)=0,
$$

which yields $a_{i j}$. Summing over $k=l$ in (22) gives an equation which is just the time derivative of (37).

We still have information remaining in equation (22), however. The remainder of equation (22) is

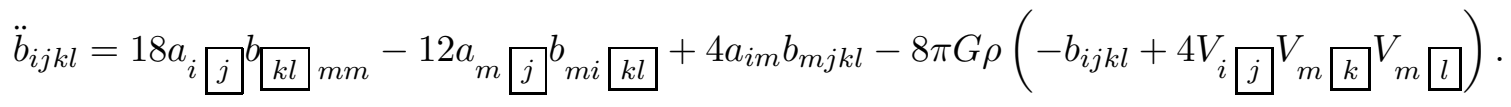

From this equation the traceless part of $b_{i j k l}$ can be determined.

Consider equation (23). The trace is given by

$$
\begin{aligned}
& 2 c_{k k l l}-16 a a_{k k}+48 \pi G(2 \rho a+p) \\
& \quad+c^{-2}\left(-\ddot{c}_{k k}-b_{k l} b_{k l}+4 a_{k k} c_{l l}-8 a_{k l} c_{k l}-24 \dot{a}^{2}+4 \pi G\left[5 \rho c_{k k}-3 a p\right]\right)=0 .
\end{aligned}
$$

\footnotetext{
${ }^{3} b_{k k}$ of course being given by the harmonic gauge conditions.
} 
It can be shown that, when combined with (19), this equation is just (31) again and thus is redundant. Now consider the symmetric piece

$$
\begin{aligned}
2 c_{i j k k}-\frac{2}{3} c_{m m k k}-16 a a_{i j}+\frac{16}{3} a a_{k k} \delta i j+c^{-2}\left\{-\ddot{c}_{i j}+\frac{1}{3} \ddot{c}_{k k} \delta_{i j}+2 b_{i k} b_{j k}-\frac{2}{3} b_{k l} b_{k l} \delta_{i j}\right. \\
\left.+4 a_{i j} c_{k k}-\frac{4}{3} a_{k k} c_{l l} \delta_{i j}-4 a_{i k} c_{j k}-4 a_{j k} c_{i k}+\frac{8}{3} a_{k l} c_{k l} \delta_{i j}+8 \pi G \rho\left[c_{i j}-\frac{1}{3} c_{k k} \delta_{i j}\right]\right\}=0
\end{aligned}
$$

which can be used in equations (34) and (35) above to define $c_{i j}$. There is no antisymmetric equation.

We have seen that equations (36) and (37) give $a_{i j}(t)$. The harmonic gauge conditions can be used to determine $b_{k k}$, and equations (34) and (35) give the trace-free part of $b_{i j}$ We may then determine $b_{i j k l}$ in the following manner: Use the harmonic gauge conditions to obtain $b_{k k i j}$ (recall that there is symmetry in the last three indices). Then use Eq. (38) to obtain the remaining $b_{i j k l}$ 's. The $c_{i j}$ 's come from equation (40). To solve for $c_{i j k l}$ use the harmonic gauge conditions to find $c_{i k k j}$ (where there is symmetry in the first two and second two indices) and Eq. (24). Finally, Equation (25) then provides $c_{i j k l m n}$.

Thus, equations (20), (21), (22), (23) and (25) determine the unknowns $a_{i j}, b_{i j k l}, b_{i j}, c_{i j k l m n}$, $c_{i j k l}$ and $c_{i j}$, and the set of equations (19), (29), (31), now contain only the eleven unknowns $\theta$, $\omega_{i j}, \sigma_{i j}, \rho$ and $A(t)$. This means that the equations can be solved for all the unknowns uniquely.

Therefore the post-Newtonian approximation provides a well-posed, closed, complete system. Thus, the post-Newtonian approximation produces a set of cosmological equations which are more in the spirit of the Bianchi types of general relativity.

\section{Newtonian Theory and the Post-Newtonian Approximation of Rotation-free Anisotropic Homogeneous Cosmologies}

\subsection{Rotation-free Anisotropic Homogeneous Newtonian Cosmology}

We first like to examine the Newtonian approximation of cosmology with vanishing rotation and diagonal shear, $\sigma_{i j}=\operatorname{diag}\left(\sigma_{11}, \sigma_{22},-\sigma_{11}-\sigma_{22}\right)$. The Newtonian approximation (Eqs. (41) and (6) to (9)) is then given by

$$
\begin{aligned}
a_{i i} & =4 \pi G \rho, \\
\dot{\rho}+\rho \theta & =0, \\
\dot{\theta} & =4 \pi G \rho-\frac{1}{3} \theta^{2}-2 \sigma^{2}, \\
\dot{\sigma}_{i j} & =a_{i j}-\frac{2}{3} \theta \sigma_{i j}-\sigma_{i k} \sigma_{k j}+\delta_{i j}\left(\frac{2}{3} \sigma^{2}-\frac{1}{3} a_{k k}\right) .
\end{aligned}
$$

For $i \neq j$, equation (44) yields

$$
a_{i j}=0, \quad i \neq j
$$

The diagonal elements of the shear obey the differential equation (44, $i=j$, no summation over $i$ )

$$
\dot{\sigma}_{i i}=a_{i i}-\frac{2}{3} \theta \sigma_{i i}-\Sigma_{k} \sigma_{i k} \sigma_{k i}+\frac{2}{3} \sigma^{2}-\frac{1}{3} \Sigma_{k} a_{k k} .
$$


Summing over $i$ just yields the definition of $\sigma^{2}$

$$
2 \sigma^{2}=\sigma_{i j} \sigma_{j i}=2\left(\sigma_{11}^{2}+\sigma_{22}^{2}+\sigma_{11} \sigma_{22}\right) \text {. }
$$

Thus, (46) provides at most two independent equations. Introducing $\theta=3 \frac{\dot{R}}{R}$ the set of equations can be written as

$$
\begin{aligned}
\frac{\ddot{R}}{R} & =\frac{4}{3} \pi G \rho_{0} R^{-3}-\frac{2}{3}\left(\sigma_{11}^{2}+\sigma_{22}^{2}+\sigma_{11} \sigma_{22}\right), \\
\rho & =\rho_{0} R^{-3} \\
a_{11} & =\dot{\sigma}_{11}+2 \frac{\dot{R}}{R} \sigma_{11}+\frac{1}{3} \sigma_{11}^{2}-\frac{2}{3}\left(\sigma_{22}^{2}+\sigma_{11} \sigma_{22}\right)+\frac{4}{3} \pi G \rho_{0} R^{-3}, \\
a_{22} & =\dot{\sigma}_{22}+2 \frac{\dot{R}}{R} \sigma_{22}+\frac{1}{3} \sigma_{22}^{2}-\frac{2}{3}\left(\sigma_{11}^{2}+\sigma_{11} \sigma_{22}\right)+\frac{4}{3} \pi G \rho_{0} R^{-3}, \\
a_{33} & =4 \pi G \rho_{0} R^{-3}-a_{11}-a_{22},
\end{aligned}
$$

where $\rho_{0}$ is a constant.

Consider the structure of these five equations: If we provide the functions $\sigma_{11}$ and $\sigma_{22}$ for all time, then the theory has a well-posed initial value problem for the variables $R(t), \rho(t)$, $a_{11}(t), a_{22}(t)$ and $a_{33}(t)$. Equation (48) is the Raychaudhuri equation (obtained from (43)), and its solution provides $R(t)$. From Eq. (49) (which comes from (42)) the density $\rho(t)$ can be extracted which, once an equation of state is provided, gives $p(t)$. Equations (50) and (51) (from (44)) give $a_{11}$ and $a_{22}$, and from (52) (or (41)), which is the Poisson equation, $a_{33}$ can be extracted.

Thus, for a homogeneous anisotropic cosmology in Newtonian theory, there is an initial value problem provided we supply two functions for all time. We have seen that the post-Newtonian theory is able to give as many equations as unknowns. So there is no need to specify any functions of time. Actually, there is no need to (arbitrarily) specify any of the unknown functions in order to obtain an initial value problem. We will revisit the Newtonian theory shortly, when we will consider the Bianchi I metric as a specific example of a homogeneous anisotropic cosmology with vanishing rotation.

\subsection{The Homogeneous Bianchi I Universe}

\subsubsection{The Metric}

Now we would like to study Newtonian theory and the post-Newtonian approximation as applied to a specific example of an anisotropic homogeneous cosmology. To this end we consider the homogeneous Bianchi I models - i.e. the Heckmann-Schücking solutions of general relativity. The metric of the general Bianchi I universe has the form

$$
d s^{2}=-d x_{0}^{2}+R_{i k} R_{j k} d x_{i} d x_{j}, \quad \text { where } x_{0}=c t \quad \text { with } R_{i j}=\operatorname{diag}\left(R_{11}, R_{22}, R_{33}\right) .
$$

To proceed further, we need to write the Bianchi I metric into a form off which the potentials $\phi, \zeta_{i}$ and $\phi_{i j}$ can be read. To do so, we consider the following transformation

$$
x_{o}=T c+\tau c^{-1}+\tau^{\prime} c^{-3},
$$

\footnotetext{
${ }^{4}$ Not to be confused with the Heckmann-Schücking cosmology of Newtonian theory.
} 


$$
x_{i}=R_{i j}^{-1} X_{j}+\chi_{i} c^{-2}+\chi_{i}^{\prime} c^{-4}
$$

with

$$
\begin{aligned}
\tau & =A(t)+A_{i j}(t) X_{i j}, \\
\tau^{\prime} & =B_{i j}(t) X_{i j}+B_{i j k l}(t) X_{i j k l}, \\
\chi_{i} & =C_{i j}(t) X_{j}+C_{i j k l}(t) X_{j k l}, \\
\chi_{i}^{\prime} & =D_{i j k l}(t) X_{j k l}+D_{i j k l m n}(t) X_{j k l m n},
\end{aligned}
$$

where $X_{i j} \equiv X_{i} X_{j}$ and similar for $X_{i j k}$ etc. Throughout the remainder of the paper we assume: $\dot{A} \ll c^{2}$ to ensure convergence of the expansion in $c^{-2}$. $A(t), A_{i j}(t), B_{i j}(t), B_{i j k l}(t), C_{i j}(t)$, $C_{i j k l}(t), D_{i j k l}(t)$ and $D_{i j k l m n}(t)$ are arbitrary functions of time. In these coordinates the metric reads

$$
\begin{aligned}
& d s^{2}=c^{2} d T^{2}\left[-1+c^{-2}\left(-2 \dot{A}-2 \dot{A}_{i j} X_{i j}+\left(R^{-2} \dot{R}^{2}\right)_{i j} X_{i j}\right)\right. \\
& +c^{-4}\left\{\left(\dot{A}+\dot{A}_{i j} X_{i j}\right)^{2}-2 \dot{B}_{i j} X_{i j}-2 \dot{B}_{i j k l} X_{i j k l}-2 \dot{R}_{i m} \dot{C}_{m j k l} X_{i j k l}-2 \dot{R}_{j l} \dot{C}_{i l} X_{i j}\right. \\
& \left.\left.-2 \dot{A}\left(\dot{R}^{2} R^{-2}\right)_{i j} X_{i j}-2 \dot{A}_{k l}\left(\dot{R}^{2} R^{-2}\right)_{i j} X_{i j k l}\right\}+\mathcal{O}\left(c^{-6}\right)\right] \\
& +c d T d X_{i}\left[c^{-1}\left(-4 A_{i j} X_{j}-2\left(\dot{R} R^{-1}\right)_{i j} X_{j}\right)\right. \\
& +c^{-3}\left(-8 \dot{A} A_{i j} X_{j}-8 A_{i j} \dot{A}_{k l} X_{j k l}-4 B_{i j} X_{j}-B_{i j k l} X_{j k l}+4 A_{i l}\left(\dot{R}^{2} R^{-2}\right)_{j k} X_{j k l}\right. \\
& +2 \dot{R}_{j l} C_{i l} X_{j}+2 R_{i l} \dot{C}_{l j}-6 \dot{R}_{j m} C_{m i k l} X_{j k l}+2 R_{i m} \dot{C}_{m j k l} X_{j k l} \\
& \left.\left.+2 \dot{A}(\dot{R} R)_{i j} X_{j}+2 \dot{A}_{k l}(\dot{R} R)_{i j} X_{j k l}\right)+\mathcal{O}\left(c^{5}\right)\right] \\
& +d X_{i} d X_{j}\left[\delta_{i j}+c^{-2}\left(-4 A_{i k} A_{j l} X_{k l}+4 A_{j k}(\dot{R} R)_{i l} X_{k l}+2 R_{i k} C_{j k}+6 R_{i m} C_{m j k l} X_{k l}\right)\right. \\
& +c^{-4}\left(-8 \dot{A} A_{i k} A_{j l} X_{k l}-8 \dot{A}_{m n} A_{i k} A_{j l} X_{k l m n}-8 B_{j k} A_{i l} X_{k l}-16 B_{i k l m} A_{j n} X_{k l m n}\right. \\
& +6 R_{i m} D_{m j k l} X_{k l}+10 R_{i p} D_{p j k l m n} X_{k l m n}+R_{m n}^{2} C_{i m} C_{j n}+9 R_{i p} R_{j q} C_{p i k l} C_{q j m n} X_{k l m n} \\
& +3 R_{m n}^{2} C_{i m} C_{n j k l} X_{k l}+3 R_{m k} R_{k n} C_{j n} C_{m i k l} X_{k l}+4\left(\dot{R}^{2} R^{-2}\right)_{k l} A_{i m} A_{j n} X_{k l m n} \\
& +4 A_{j l} \dot{R}_{k m} C_{m i} X_{k l}+12 A_{j n} \dot{R}_{k p} C_{p i l m} X_{k l m n}+4 A_{j m} R_{i l} \dot{C}_{k l} X_{k m}+4 A_{j n} R_{p i} \dot{C}_{p k l m} X_{k l m n} \\
& +4 \dot{A}\left(\dot{R} R^{-1}\right)_{i k} A_{j l} X_{k l}+4\left(\dot{R} R^{-1}\right)_{i k} \dot{A}_{m n} A_{j l} X_{k l m n}+4\left(\dot{R} R^{-1}\right)_{i k} B_{j l} X_{k l} \\
& \left.\left.+8\left(\dot{R} R^{-1}\right)_{i k} B_{j l m n} X_{k l m n}\right)+\mathcal{O}\left(c^{-6}\right)\right] \text {. }
\end{aligned}
$$

It can be seen from (10) that there are no terms of order $c^{-1}$, thus

$$
A_{i j}=-\frac{1}{2}\left(\dot{R} R^{-1}\right)_{i j}
$$

Also, from (10) we see that the $c^{-2}$ terms in $g_{00}$ and $g_{i j}$ must be identical. Hence,

$$
\begin{aligned}
C_{i j} & =\dot{A} R_{i j}^{-1}, \\
C_{i j k l} & =\frac{1}{4}\left[\left(\dot{R} R^{-2}\right)_{i l}\left(\dot{R} R^{-1}\right)_{j k}+\left(\dot{R} R^{-2}\right)_{i k}\left(\dot{R} R^{-1}\right)_{j l}\right]+\frac{1}{6} R_{i j}^{-1}\left(\ddot{R} R^{-1}\right)_{k l} .
\end{aligned}
$$


We may now read off the potentials $\phi, \zeta_{i}$ and $\phi_{i j}$ from the metric (54). With the help of the harmonic gauge conditions (11) and (12) we are able to write down the functions $a(t), a_{i j}(t)$ etc. which appear in the ansätze (18):

$$
\begin{aligned}
& a(t)=\dot{A}, \\
& a_{i j}(t)=-\frac{1}{2}\left(\ddot{R} R^{-1}\right)_{i j}, \\
& b_{i j}(t)=-4 B_{i j}+6 \dot{A}\left(\dot{R} R^{-1}\right)_{i j}-2 \ddot{A} \delta_{i j}
\end{aligned}
$$

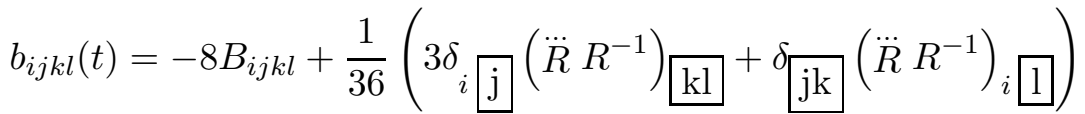

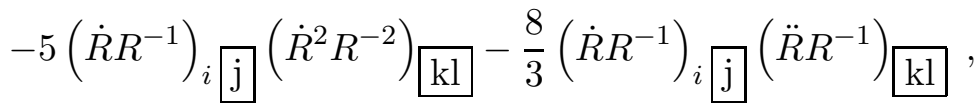

$$
\begin{aligned}
& c_{i j}(t)=2 \dot{A}^{2} \delta_{i j} \\
& c_{i j k l}(t)=-\delta_{i j}\left(\dot{B}_{k l}+\dot{B}_{l k}\right)-3 \delta_{i j} \dot{A}\left(\dot{R}^{2} R^{-2}\right)_{k l}-2 \delta_{i j} \dot{A}\left(\ddot{R} R^{-1}\right)_{k l}+2 \delta_{i j} \ddot{A}\left(\dot{R} R^{-1}\right)_{k l} \\
& +\frac{1}{2} \delta_{i k}\left(\dot{R} R^{-1}\right)_{j l}+\frac{1}{2} \delta_{j k}\left(\dot{R} R^{-1}\right)_{i l}+\frac{1}{2} \delta_{i l}\left(\dot{R} R^{-1}\right)_{j k}+\frac{1}{2} \delta_{j l}\left(\dot{R} R^{-1}\right)_{i k} \\
& +3 R_{i m} D_{m j k l}+3 R_{j m} D_{m i k l}+2 B_{i k}\left(\dot{R} R^{-1}\right)_{j l}+2 B_{i l}\left(\dot{R} R^{-1}\right)_{j k}+2 B_{j k}\left(\dot{R} R^{-1}\right)_{i l} \\
& +2 B_{j l}\left(\dot{R} R^{-1}\right)_{i k}-\frac{11}{4} \dot{A}\left(\dot{R} R^{-1}\right)_{i k}\left(\dot{R} R^{-1}\right)_{j l}-\frac{11}{4} \dot{A}\left(\dot{R} R^{-1}\right)_{i l}\left(\dot{R} R^{-1}\right)_{j k} \\
& -\frac{3}{4} \dot{A}\left(\dot{R} R^{-1}\right)_{i k}\left(\ddot{R} R^{-1}\right)_{j l}-\frac{3}{4} \dot{A}\left(\dot{R} R^{-1}\right)_{i l}\left(\ddot{R} R^{-1}\right)_{j k} \\
& -\frac{3}{4} \dot{A}\left(\dot{R} R^{-1}\right)_{j k}\left(\ddot{R} R^{-1}\right)_{i l}-\frac{3}{4} \dot{A}\left(\dot{R} R^{-1}\right)_{j l}\left(\ddot{R} R^{-1}\right)_{i k} \text {, } \\
& c_{i j k l m n}(t)=-2 \delta_{i j} \dot{B} \mathrm{klmn}^{+}+\frac{1}{4}\left(\dot{R}^{2} R^{-2}\right)_{\mathrm{kl}}\left(\dot{R}^{2} R^{-2}\right)_{\mathrm{mn}} \delta_{i j} \\
& -\frac{1}{6} \delta_{i j}\left(\ddot{R} R^{-1}\right)_{\mathrm{kl}}\left(\dot{R}^{2} R^{-2}\right)_{\mathrm{mn}}+\frac{1}{2} \delta_{i j}\left(\ddot{R} R^{-1}\right)_{\mathrm{kl}}\left(\ddot{R} R^{-1}\right)_{\mathrm{mn}} \\
& +2 \delta_{i j}\left(\dot{R} R^{-1}\right)_{\mathrm{kl}}\left(\dot{R}^{3} R^{-3}\right)_{\mathrm{mn}}-\frac{2}{3}\left(\dot{R} R^{-1}\right)_{\mathrm{kl}}\left(\ddot{R} \dot{R} R^{-2}\right)_{\mathrm{mn}} \\
& -\frac{1}{3} \delta_{i j}\left(\dot{R} R^{-1}\right)_{\mathrm{kl}}\left(\dddot{R} R^{-1}\right)_{\mathrm{mn}}+5 R_{i o} D_{o j k l m n}+5 R_{j o} D_{o i k l m n} \\
& +8 B_{i \mathrm{klm}}\left(\dot{R} R^{-1}\right)_{j \mathrm{n}}+8 B_{j \mathrm{klm}}\left(\dot{R} R^{-1}\right)_{i \mathrm{n}} \\
& -\frac{1}{6}\left(\dot{R} R^{-1}\right)_{i \mathrm{k}}\left(\dot{R} R^{-1}\right)_{j \mathrm{l}}\left(\ddot{R} R^{-1}\right)_{\mathrm{mn}} \\
& +\frac{25}{4}\left(\dot{R} R^{-1}\right)_{i} \mathrm{k}\left(\dot{R} R^{-1}\right)_{j} \mathrm{l}\left(\dot{R}^{2} R^{-2}\right)_{\mathrm{mn}} \\
& +\frac{1}{6} \delta_{i \mathrm{k}}\left(\dot{R} R^{-1}\right)_{{ }_{\mathrm{jl}}}\left(\ddot{R} \dot{R} R^{-2}\right)_{\mathrm{mn}}-\frac{1}{6} \delta_{i \mathrm{k}}\left(\dot{R} R^{-1}\right)_{\mathrm{jl}}\left(\dddot{R} R^{-1}\right)_{\mathrm{mn}} \\
& +\frac{1}{6} \delta_{j \mathrm{k}}\left(\dot{R} R^{-1}\right)_{\mathrm{il}}\left(\ddot{R} \dot{R} R^{-2}\right)_{\mathrm{mn}}-\frac{1}{6} \delta_{j \mathrm{k}}\left(\dot{R} R^{-1}\right)_{\mathrm{il}}\left(\dddot{R} R^{-1}\right)_{\mathrm{mn}} \\
& +\left(\dot{R}^{2} R^{-2}\right)_{i \mathrm{k}}\left(\dot{R} R^{-1}\right)_{j \mathrm{l}}\left(\dot{R} R^{-1}\right)_{\mathrm{Ln}}+\left(\dot{R}^{2} R^{-2}\right)_{j \mathrm{k}}\left(\dot{R} R^{-1}\right)_{i \mathrm{l}}\left(\dot{R} R^{-1}\right)_{\mathrm{mn}} \\
& -\frac{1}{2}\left(\ddot{R} R^{-1}\right)_{i \mathrm{k}}\left(\dot{R} R^{-1}\right)_{j} \mathrm{l}\left(\dot{R} R^{-2}\right)_{\mathrm{mn}}-\frac{1}{2}\left(\ddot{R} R^{-1}\right)_{j \mathrm{k}}\left(\dot{R} R^{-1}\right)_{i}\left[\mathrm{l}\left(\dot{R} R^{-2}\right)_{\mathrm{mn}}\right. \text {. }
\end{aligned}
$$


The Heckmann-Schücking solution has $R_{i j}=\operatorname{diag}\left(R_{11}, R_{22}, R_{33}\right)$ such that

$$
\begin{aligned}
& R_{11}=\left(t-t_{0}\right)^{p_{1}}\left(t-t_{1}\right)^{q_{1}}, \\
& R_{22}=\left(t-t_{0}\right)^{p_{2}}\left(t-t_{1}\right)^{q_{2}}, \\
& R_{33}=\left(t-t_{0}\right)^{p_{3}}\left(t-t_{1}\right)^{q_{3}}
\end{aligned}
$$

with

$$
\Sigma_{i} p_{i}=\Sigma_{i} p_{i}^{2}=1
$$

where

$$
q_{i}=\frac{2}{3}-p_{i}
$$

such that

$$
\Sigma_{i} q_{i}=\Sigma_{i} q_{i}^{2}=1
$$

These conditions can be fulfilled by the following parameterisation for $p_{1}$ to $p_{3}$ and $q_{1}$ to $q_{3}$ :

$$
\begin{aligned}
& p_{1}:=u, \\
& p_{2}:=\frac{1-u}{2}+\frac{1}{2} \sqrt{1+2 u-3 u^{2}}, \\
& p_{3}:=\frac{1-u}{2}-\frac{1}{2} \sqrt{1+2 u-3 u^{2}}, \\
& q_{1}:=\frac{2}{3}-u \\
& q_{2}:=\frac{1+3 u}{6}-\frac{1}{2} \sqrt{1+2 u-3 u^{2}}, \\
& q_{3}:=\frac{1+3 u}{6}+\frac{1}{2} \sqrt{1+2 u-3 u^{2}} .
\end{aligned}
$$

\subsubsection{The Heckmann-Schücking Solution of General Relativity and Newtonian Theory}

The Bianchi I model is rotation-free and so the Newtonian theory is just given by equations (48) to (52). From (55) we see that $a_{i j}=-\frac{1}{2} \frac{\ddot{R}_{i k}}{R_{j k}}$. Define $R(t)$ such that

$$
\theta=3 \frac{\dot{R}}{R}=\frac{\left(R_{11} R_{22} R_{33}\right)}{R_{11} R_{22} R_{33}}=\frac{2 t-t_{0}-t_{1}}{\left(t-t_{0}\right)\left(t-t_{1}\right)} .
$$

The parameters to be determined are $u, t_{0}, t_{1}$ and $\rho_{0}$, and the variables are $\rho, \sigma_{11}, \sigma_{22}$.

First consider equations (50) and (51) which using the parametrisation (62) become:

$$
\begin{aligned}
\sigma_{11}+\frac{2}{3} \sigma_{11} \frac{2 t-t_{0}-t_{1}}{\left(t-t_{0}\right)\left(t-t_{1}\right)}+\frac{1}{3} \sigma_{11}^{2}-\frac{2}{3}\left(\sigma_{22}^{2}\right. & \left.+\sigma_{11} \sigma_{22}\right)+\frac{4}{3} \pi G \rho \frac{1}{\left(t-t_{0}\right)\left(t-t_{1}\right)}= \\
& -\frac{u(u-1)}{2\left(t-t_{0}\right)^{2}}+\frac{(2-3 u)(1+3 u)}{18\left(t-t_{1}\right)^{2}}-\frac{u(2-3 u)}{3\left(t-t_{0}\right)\left(t-t_{1}\right)}
\end{aligned}
$$

and

$\sigma_{22}+\frac{2}{3} \sigma_{22} \frac{2 t-t_{0}-t_{1}}{\left(t-t_{0}\right)\left(t-t_{1}\right)}+\frac{1}{3} \sigma_{22}^{2}-\frac{2}{3}\left(\sigma_{11}^{2}+\sigma_{11} \sigma_{22}\right)+\frac{4}{3} \pi G \rho \frac{1}{\left(t-t_{0}\right)\left(t-t_{1}\right)}=$ 


$$
\begin{aligned}
& -\frac{\left(1-u+\sqrt{1+2 u-3 u^{2}}\right)\left(1+u-\sqrt{1+2 u-3 u^{2}}\right)}{8\left(t-t_{0}\right)^{2}} \\
& +\frac{\left(1+3 u-3 \sqrt{1+2 u-3 u^{2}}\right)\left(5-u+3 \sqrt{1+2 u-3 u^{2}}\right)}{72\left(t-t_{1}\right)^{2}} \\
& -\frac{\left(1-u+\sqrt{1+2 u-3 u^{2}}\right)\left(1+3 u-3 \sqrt{1+2 u-3 u^{2}}\right)}{12\left(t-t_{0}\right)\left(t-t_{1}\right)}
\end{aligned}
$$

Once the parameters $u, t_{0}$ and $t_{1}$ are known we are able to solve these two equations for $\sigma_{11}$ and $\sigma_{22}$. 5 To proceed further, consider Eq. (48) which is a Raychaudhuri-like equation and may be written as

$$
\frac{4}{3} \pi \rho_{0} \frac{1}{\left(t-t_{0}\right)\left(t-t_{1}\right)}-\frac{2}{3}\left(\sigma_{11}^{2}+\sigma_{22}^{2}+\sigma_{11} \sigma_{22}\right)=\frac{1}{3\left(\left(t-t_{0}\right)\left(t-t_{1}\right)\right)^{\frac{5}{3}}}-\frac{2}{9} \frac{2 t-t_{0}-t_{1}}{\left(\left(t-t_{0}\right)\left(t-t_{1}\right)\right)^{\frac{8}{3}}} .
$$

If $\sigma_{11}$ and $\sigma_{22}$ are known then this equation relates the two constants $t_{0}$ and $t_{1}$. The Poisson equation (52) simply defines $\rho_{0}$,

$$
\rho_{0}=\frac{1}{12 \pi G}
$$

Finally, we can write down (49) as

$$
\rho=\rho_{0} \frac{1}{\left(t-t_{0}\right)\left(t-t_{1}\right)},
$$

which determines $\rho(t)$. Therefore the shear variables, $\sigma_{11}$ and $\sigma_{22}$, and the density $\rho$ are now expressed in terms of two parameters with the initial condition $\rho(t=0)$ specifying one of them.

Thus, the Newtonian theory in the homogeneous, anisotropic rotation-free case can be solved, provided we arbitrarily fix the parameter $u$ for all time. However, varying the equation of state will have no effect on the solutions for the density, since the pressure in no way enters the dynamics, as was the case in [7] with the FRW cosmology.

\subsubsection{The Post-Newtonian Approximation of the Homogeneous Bianchi I Universe}

The post-Newtonian theory for the homogeneous anisotropic rotation-free case is given by equations (20), (21), (22), (25), (24, $m \neq n)$ and (23) which may be solved for the unknowns $a_{i j}$, $b_{i j}, b_{i j k l}, c_{i j}, c_{i j k l}$ and $c_{i j k l m n}$ as we have shown in section 2. The set of equations (19), (29), and (31) then determine the eight unknowns $\theta, \sigma_{i j}, \rho$ and $A(t)$ (or $a(t)$ ). Vanishing rotation imposes extra symmetry on the equations. Therefore many become redundant and there are a great many simplifications in what does remain. Also we can use the potentials (55) for further simplification. Clearly $a_{i j}=0$ for $i \neq j$, since $a_{i j}=-\frac{1}{2} \frac{\ddot{R}_{i k}}{R_{j k}}$, with $R_{i j}=\operatorname{diag}\left(R_{11}, R_{22}, R_{33}\right)$.

Using the parametrisation (62) the Poisson-like equation (19) becomes

$$
\frac{1}{\left(t-t_{0}\right)\left(t-t_{1}\right)}=6 \pi G \rho+c^{-2}\left\{24 \pi G \rho+\frac{3}{2} \dddot{A}\right\}
$$

which can be used to determine the potential $A(t)$.

The Friedmann-like equation (29), as usual, provides $\rho(t)$ :

$\dot{\rho}+\rho \frac{2 t-t_{0}-t_{1}}{\left(t-t_{0}\right)\left(t-t_{1}\right)}+c^{-2}\left\{-4 \dot{\rho} \dot{A}-2 \rho \ddot{A}+p \frac{2 t-t_{0}-t_{1}}{\left(t-t_{0}\right)\left(t-t_{1}\right)}+\frac{\ddot{A}}{2 \pi G} \frac{1}{\left(t-t_{0}\right)\left(t-t_{1}\right)}\right\}=0$.

\footnotetext{
${ }^{5}$ The condition $\sigma_{11}+\sigma_{22}+\sigma_{33}=0$ determines $\sigma_{33}$.
} 
The Bianchi identity (31) can be decomposed into a trace part, symmetric trace-less part and an antisymmetric part. The trace yields the Raychaudhuri-like equation

$$
\begin{aligned}
& \frac{6\left(t-t_{0}\right)\left(t-t_{1}\right)-2\left(2 t-t_{0}-t_{1}\right)^{2}}{\left(t-t_{0}\right)^{2}\left(t-t_{1}\right)^{2}}+4 \pi G\left(\rho+3 p c^{-2}\right)-\sigma^{2} \\
& -c^{-} 2\left\{\frac{\dot{A}}{9}\left[\frac{124\left(2 t-t_{0}-t_{1}\right)^{2}}{\left(t-t_{0}\right)^{2}\left(t-t_{1}\right)^{2}}-\frac{14}{\left(t-t_{0}\right)\left(t-t_{1}\right)}\right]\right. \\
& +\frac{22}{3} \ddot{A} \frac{2 t-t_{0}-t_{1}}{\left(t-t_{0}\right)\left(t-t_{1}\right)}+\frac{123}{8} \dddot{A}+\sigma^{2}\left[-\frac{5}{3} \dot{A}+\frac{3}{8} c_{k k l l}\left(t-t_{0}\right)\left(t-t_{1}\right)\right] \\
& +c_{k k l l}\left(\frac{63}{16}-\frac{1}{2} \frac{\left(2 t-t_{0}-t_{1}\right)^{2}}{\left(t-t_{0}\right)\left(t-t_{1}\right)}\right)+\dot{c}_{k k l l}\left(2 t-t_{0}-t_{1}\right)-\frac{9}{4} b_{k} l\left(b_{k l m m}+b_{l k m m}\right) \\
& -96 \dot{A}\left[\left(\frac{u(3 u-2)}{3\left(t-t_{0}\right)\left(t-t_{1}\right)}-\frac{u(u-1)}{2\left(t-t_{1}\right)^{2}}+\frac{(2-3 u)(3 u+1)}{18\left(t-t_{0}\right)^{2}}\right)^{2}\right. \\
& +\left\{\frac{\left(u-1-\sqrt{1+2 u-3 u^{2}}\right)\left(-u-1+\sqrt{1+2 u-3 u^{2}}\right)}{4\left(t-t_{0}\right)\left(t-t_{1}\right)}\right. \\
& +\frac{\left(1-u+\sqrt{1+2 u-3 u^{2}}\right)\left(u+1-\sqrt{1+2 u-3 u^{2}}\right)}{8\left(t-t_{1}\right)^{2}} \\
& \left.-\frac{\left(3 u+1-3 \sqrt{1+2 u-3 u^{2}}\right)\left(3 u-5-3 \sqrt{1+2 u-3 u^{2}}\right)}{72\left(t-t_{0}\right)^{2}}\right\}^{2} \\
& +\left\{\frac{\left(u-1+\sqrt{1+2 u-3 u^{2}}\right)\left(3 u+1+3 \sqrt{1+2 u-3 u^{2}}\right)}{12\left(t-t_{0}\right)\left(t-t_{1}\right)}\right. \\
& +\frac{\left(1-u-\sqrt{1+2 u-3 u^{2}}\right)\left(u+1-\sqrt{1+2 u-3 u^{2}}\right)}{8\left(t-t_{1}\right)^{2}} \\
& \left.\left.-\frac{\left(3 u+1+3 \sqrt{1+2 u-3 u^{2}}\right)\left(3 u-5+3 \sqrt{1+2 u-3 u^{2}}\right)}{72\left(t-t_{0}\right)^{2}}\right\}^{2}\right] \\
& +\frac{1}{24} \dot{b}_{11}\left[-6 u(3 u-2)+\frac{9 u(u-1)\left(t-t_{0}\right)}{t-t_{1}}+\frac{(3 u-2)(3 u+1)\left(t-t_{1}\right)}{t-t_{0}}\right] \\
& +\frac{1}{96} \dot{b}_{22}\left[6\left(1-u+\sqrt{1+2 u-3 u^{2}}\right)\left(1+3 u-3 \sqrt{1+2 u-3 u^{2}}\right)\right. \\
& -\frac{9\left(1-u+\sqrt{1+2 u-3 u^{2}}\right)\left(1+u-\sqrt{1+2 u-3 u^{2}}\right)\left(t-t_{0}\right)}{t-t_{1}} \\
& \left.+\frac{\left(1+3 u-3 \sqrt{1+2 u-3 u^{2}}\right)\left(-5+3 u-3 \sqrt{1+2 u-3 u^{2}}\right)\left(t-t_{1}\right)}{t-t_{0}}\right] \\
& +\frac{1}{96} \dot{b}_{33}\left[6\left(1-u-\sqrt{1+2 u-3 u^{2}}\right)\left(1+3 u+3 \sqrt{1+2 u-3 u^{2}}\right)\right. \\
& -\frac{9\left(1-u-\sqrt{1+2 u-3 u^{2}}\right)\left(1+u+\sqrt{1+2 u-3 u^{2}}\right)\left(t-t_{0}\right)}{t-t_{1}} \\
& \left.+\frac{\left(1+3 u+3 \sqrt{1+2 u-3 u^{2}}\right)\left(-5+3 u+3 \sqrt{1+2 u-3 u^{2}}\right)\left(t-t_{1}\right)}{t-t_{0}}\right]
\end{aligned}
$$




$$
\begin{aligned}
& +\frac{1}{6} b_{11}\left[\frac{3 u(2-3 u)\left(2 t-t_{0}-t_{1}\right)}{\left(t-t_{0}\right)\left(t-t_{1}\right)}+\frac{9 u(u-1)\left(t-t_{0}\right)}{\left(t-t_{1}\right)^{2}}-\frac{(2-3 u)(3 u+1)\left(t-t_{1}\right)}{\left(t-t_{0}\right)^{2}}\right] \\
& +\frac{1}{48} b_{22}\left[\frac{6\left(-1+u-\sqrt{1+2 u-3 u^{2}}\right)\left(1+3 u-3 \sqrt{1+2 u-3 u^{2}}\right)\left(2 t-t_{0}-t_{1}\right)}{\left(t-t_{0}\right)\left(t-t_{1}\right)}\right. \\
& +\frac{12\left(-1-u+\sqrt{1+2 u-3 u^{2}}\right)\left(1+u-\sqrt{1+2 u-3 u^{2}}\right)\left(t-t_{0}\right)}{\left(t-t_{1}\right)^{2}} \\
& \left.+\frac{\left(1+3 u-3 \sqrt{1+2 u-3 u^{2}}\right)\left(-5+u-3 \sqrt{1+2 u-3 u^{2}}\right)\left(t-t_{1}\right)}{\left(t-t_{0}\right)^{2}}\right] \\
& +\frac{1}{48} b_{33}\left[\frac{6\left(-1+u+\sqrt{1+2 u-3 u^{2}}\right)\left(1+3 u+3 \sqrt{1+2 u-3 u^{2}}\right)\left(2 t-t_{0}-t_{1}\right)}{\left(t-t_{0}\right)\left(t-t_{1}\right)}\right. \\
& \left.\left.+\frac{12\left(1-u-\sqrt{1+2 u-3 u^{2}}\right)\left(1+u+\sqrt{1+2 u-3 u^{2}}\right)\left(t-t_{0}\right)}{\left(t-t_{1}\right)^{2}}\right]\right\}=0, \\
& \left.\left.-\frac{\left(1+3 u+3 \sqrt{1+2 u-3 u^{2}}\right)\left(-5+u+3 \sqrt{1+2 u-3 u^{2}}\right)\left(t-t_{1}\right)}{\left(t-t_{0}\right)^{2}}\right]\right)
\end{aligned}
$$

which may be used to determine $u$.

The traceless symmetric part is given by

$$
\begin{aligned}
& \dot{\sigma}_{i j}+ \frac{2}{3} \sigma_{i j} \frac{2 t-t_{0}-t_{1}}{\left(t-t_{0}\right)\left(t-t_{1}\right)}+\sigma_{i j}^{2}-\frac{2}{3} \sigma^{2} \delta_{i j}+2 a_{i j}-\frac{2}{9\left(t-t_{0}\right)\left(t-t_{1}\right)} \delta_{i j}= \\
& c^{-2}\left\{\frac{1}{12} \sigma_{i j}\left[106 \ddot{A}-56 \dot{A} \frac{2 t-t_{0}-t_{1}}{\left(t-t_{0}\right)\left(t-t_{1}\right)}+3\left(t-t_{0}\right)\left(t-t_{1}\right) \dot{c}_{k k l l}+3\left(2 t-t_{0}-t_{1}\right) c_{k k l l}\right]\right. \\
&+\frac{70}{3} \dot{A} a_{i j}+\frac{1}{8}\left(t-t_{0}\right)\left(t-t_{1}\right) c_{k k l l}\left(23 a_{i j}+2 \dot{\sigma}_{i j}\right)+\frac{4}{3} \dot{A} \dot{\sigma}_{i j} \\
&+\frac{2\left[\dot{A}+9 \ddot{A}\left(2 t-t_{0}-t_{1}\right)\right]}{27\left(t-t_{0}\right)\left(t-t_{1}\right)} \delta_{i j}-\frac{1}{8}\left(\dot{b}_{i j}+\dot{b}_{j i}\right)-\frac{23}{72} c_{k k l l} \delta_{i j} \\
&+\left(t-t_{0}\right)\left(t-t_{1}\right)\left[-3 \dddot{A} a_{i j}+9 \ddot{A} \dot{a}_{i j}+\frac{9}{2}\left(\dot{a}_{i k} b_{k j}+\dot{a}_{J k} b_{k i}\right)-\frac{7}{2} \dot{a}_{k l} b_{k l} \delta_{i j}\right. \\
&-36 a a_{i k} a_{j k}+12 a a_{k l} a_{k l} \delta_{i j}+\frac{3}{4}\left(\dot{a}_{j k} b_{i k}+\dot{a}_{i k} b_{j k}\right) \\
&-\frac{3}{4}\left(b_{i k} b_{k j l l}+b_{j k} b_{k i l l}+3 b_{k l} b_{k l i j}+3 b_{k l} b_{l k i j}-b_{k l} b_{k l m m} \delta_{i j}-b_{k l} b_{l k m m} \delta_{i j}\right) \\
&\left.\left.+\frac{3}{2}\left(a_{i k} c_{l l j k}+a_{j k} c_{l l i k}\right)-a_{k m} c_{l l k m} \delta_{i j}+3 a_{k l} c_{k l i j}\right]\right\}
\end{aligned}
$$

and may be used to find $\sigma_{11}, \sigma_{22}$ and $\sigma_{33}=-\sigma_{11}-\sigma_{22}$.

The antisymmetric piece of Eq. (31) is

$$
\begin{aligned}
4\left(\dot{a}_{i k} k j-\dot{a}_{j k} b_{k i}\right)+2\left(\dot{a}_{j k} b_{i k}-\dot{a}_{i k} b_{j k}\right. & \left.-a_{j k} \dot{b}_{i k}+a_{i k} \dot{b}_{j k}\right) \\
& =3\left(b_{i k} b_{k j l l}-b_{j k} b_{k i l l}-b_{k j} b_{i k l l}+b_{k i} b_{j k l l}\right) .
\end{aligned}
$$

These are three equations. However, with the aid of the harmonic gauge conditions it can be shown that only two of the equations are independent. These can be used to determine $t_{0}$ and $t_{1}$. 
Thus, the Heckmann-Schücking solutions of general relativity for the post-Newtonian approximation are defined by the eight variables $\rho, R_{11}, R_{22}, R_{33}, \sigma_{11}, \sigma_{22} \sigma_{33}, A(t)$, [ which are completely determined by the equations (64) to (68).

A second improvement on the Newtonian theory is that here pressure enters into the dynamics through the Raychaudhuri equation. This means that varying the equation of state varies the solutions for the density $\rho$, the cosmic scale factor $R(t)$ and the shear $\sigma(t)$. As in the FRW cosmology [7] the function of time $A(t)$ is what incorporates the pressure into the system.

\section{Newtonian Theory and the Post-Newtonian Approximation of Shear-Free Anisotropic Homogeneous Cosmologies}

\subsection{The Newtonian Shear-Free Anisotropic Homogeneous Universe}

The Heckmann-Schücking solution of section 2.1 is the Newtonian approximation of an anisotropic homogeneous cosmology. The cosmology is given by equations (41), (6) and (7) to (9) with vanishing shear. The equations reduce to

$$
\begin{array}{r}
a_{i i}=4 \pi G \rho, \\
\rho=\rho_{0} R^{-3}, \\
\omega_{i}=\omega_{i 0} R^{-2}, \\
\frac{\ddot{R}}{R}=-\frac{4 \pi G \rho_{0}}{3 R^{3}}+\frac{2 \omega_{0}^{2}}{R^{4}} .
\end{array}
$$

Recall that with the five shear functions arbitrarily set to zero we have a determined system. The final equation (72) may be integrated out to give the Heckmann-Schücking solution

$$
\dot{R}^{2}=\frac{8 \pi G \rho_{0}}{3 R}-\frac{2 \omega_{0}^{2}}{3 R^{2}}-\frac{\epsilon}{\tau_{0}^{2}}
$$

where $\epsilon= \pm 1$ or $0, \tau_{o}$ an arbitrary constant. When $\omega_{i 0}=0$ we have a shear-free and rotationfree cosmology which becomes isotropic. This is just FRW, which is the most general possible solution for homogeneity and isotropy. In this case equation (73) can be identified with the Raychaudhuri equation of the FRW cosmology.

A theorem of Ellis [i] states that in the case of shear-free dust either the expansion or the rotation must vanish. Setting $\theta=0$, we obtain the following solution for $R(t)$

$$
R=\frac{2 \omega_{o}^{2}}{8 \pi G \rho_{0}}
$$

Hence $R$ is a constant, and as long as $\omega_{0}$ is non-zero, there is no possibility of there being a singularity for $R(t)$. The solution will have minimum and maximum values for $R(t)$ but may never be zero, 10.

Next we explore the analogous case in the post-Newtonian approximation and see how far it comes in overcoming this difficulty.

\subsection{The Post-Newtonian Approximation of an Anisotropic Homogeneous Shear-free Universe}

The post-Newtonian approximation for the shear-free case is given by the field equations (20), (21), (22), (25), (24, $m \neq n$ ) and (23), along with a Poisson-like equation (19) and the Bianchi

\footnotetext{
${ }^{6}$ And, $p(t)$ which is defined through a barotropic equation of state.
} 
identities (29), (31), with $\sigma_{i j}=0$. The equations (19), (29) and (31) are eleven equations, and the variables are reduced to $\rho$ (which may be determined from (29) $), \theta$ (which may be determined from the trace of (31)), $\omega_{i j}$ (which may be determined from the antisymmetric part of (31)) and $a(t)$ (which may be determined from the Poisson-like equation (19)). Unlike in the rotation-free case where the $a_{i j}$ can be determined from the additional field equations here we will use what remains of the symmetric part of (31) to determine the $a_{i j}$ for $i \neq j$. The unknowns $b_{i j}, b_{i j k l}$, $c_{i j}, c_{i j k l}$ and $c_{i j k l m n}$ that appear in the $c^{-2}$ corrections to (19), (29) and (31) are determined from the additional field equations (20), (21), (22), (23), (24, $m \neq n)$ and (25).

The reduction in unknowns must be accompanied by redundancies otherwise the system will be overdetermined. We saw earlier that equations (36) and (37) yield $a_{i j}$. Somewhat tedious calculations show that the time derivative of the symmetric part of (21) and (24) give the symmetric part of (31) in this special case of vanishing shear. Thus, we may ignore the symmetric part of (31).

The Poisson-like equation (19) is

$$
a_{k k}=2 \pi G \rho+c^{-2}\left(-\frac{1}{4} c_{k l k l}+8 \pi G \rho a\right),
$$

which defines $a(t)$. Defining $\theta=3 \frac{R(t)}{R(t)}$ we may write the continuity equation (29) which allows us to solve for $\rho$ as $^{7}$

$$
\left(\frac{\left(\left(R_{11} R_{22} R_{33}\right)^{\frac{1}{3}}\right)}{\left(R_{11} R_{22} R_{33}\right)^{\frac{1}{3}}}\right)^{2}=\frac{8 \pi G}{3} \rho+\gamma c^{-2},
$$

where $\gamma$ is a solution of the differential equation

$$
\begin{aligned}
& \dot{\gamma}+4 \gamma \frac{\left(\left(\left(R_{11} R_{22} R_{33}\right)^{\frac{1}{3}}\right)^{\cdot \cdot}\right)}{\left(R_{11} R_{22} R_{33}\right)^{\frac{1}{3}}}+\ddot{A} \frac{\left(\left(R_{11} R_{22} R_{33}\right)^{\frac{1}{3}}\right)^{\cdot \cdot}}{\left(R_{11} R_{22} R_{33}\right)^{\frac{1}{3}}} \\
& +8 \dot{a}\left(\frac{\left(\left(R_{11} R_{22} R_{33}\right)^{\frac{1}{3}}\right)^{\cdot \cdot}\left(\left(R_{11} R_{22} R_{33}\right)^{\frac{1}{3}}\right)}{\left(R_{11} R_{22} R_{33}\right)^{\frac{2}{3}}}-\frac{\left(\left(R_{11} R_{22} R_{33}\right)^{\frac{1}{3}}\right)^{\cdots}}{\left(R_{11} R_{22} R_{33}\right)^{\frac{1}{3}}}\right)=0 .
\end{aligned}
$$

The antisymmetric part of (31) becomes

$$
\begin{aligned}
& \dot{\omega}_{i j}+ \frac{2}{3} \theta \omega_{i j}+\frac{1}{\rho} c^{-2}\left(\omega_{i j}\left(-2 \rho \dot{a}-2 \dot{\rho} a+2 \rho a \theta+\frac{1}{2} \rho b_{k k}\right)-\frac{1}{2} \rho\left(c_{i k j k}-c_{j k i k}\right)\right. \\
&+2 \rho a \dot{\omega}_{i j}-\frac{4}{3} \rho a \theta \omega_{i j}+\frac{1}{16 \pi G}\left[-\frac{8}{3} \theta \omega_{i j}-\frac{4}{3} \omega_{i j}\left(\dddot{a}+4 \dot{a} a_{k k}+4 a \dot{a}_{k k}\right)\right. \\
& \quad-\frac{4}{3}\left(\dot{\omega}_{i j}+\frac{2}{3} \theta \omega_{i j}+\frac{1}{2} \omega_{i k} \omega_{k j}-\frac{1}{2} \omega_{j k} \omega_{k i}\right)\left(\ddot{a}+4 a a_{k k}\right)-24 \omega_{i j} a_{k k} \dot{a}-4 \dot{a}_{k j} b_{k i} \\
&+4 \dot{a}_{k i} b_{k j}-4 a_{k j} \dot{b}_{k i}+4 a_{k i} \dot{b}_{k j}-15 b_{i k} b_{l l k j}+15 b_{j k} b_{l l k i}-12 a_{i k} c_{l l k j} \\
&+12 a_{j k} c_{l l k i}-4 a_{i k} c_{k l j l}+4 a_{j k} c_{k l i l}-4 a_{j k} c_{i l k l}+4 a_{i k} c_{j l k l}+8 a_{l k} c_{l i k j} \\
&\left.\left.\quad-8 a_{l k} c_{l j k i}-8 a_{l k} c_{i k l j}+8 a_{l k} c_{j k i l}\right]\right)=0,
\end{aligned}
$$

which may be solved for $\omega_{i j}$. Finally, the trace of (31) gives a Raychaudhuri-like equation which is

$$
3 \frac{\ddot{R}}{R}=4 \pi G\left(\rho+3 p c^{-2}\right)+2 \omega^{2}+c^{-2}\left(-114 a\left(\frac{\dot{R}}{R}\right)^{2}+20 \dot{a} \frac{\dot{R}}{R}+8 a a_{k k}-\frac{95}{16} \ddot{a}\right.
$$

\footnotetext{
${ }^{7}$ Which is the same as in the rotation-free case.
} 


$$
\begin{gathered}
-\frac{51}{2} c_{k l k l}+\frac{10}{3} a \omega^{2}+\frac{1}{4 \pi G \rho}\left[-6 \ddot{a}\left(\frac{\dot{R}}{R}\right)^{2}-\ddot{a} \frac{\ddot{R}}{R}-\frac{1}{3} \dddot{a}-\frac{2}{3} \ddot{a} \omega^{2}\right. \\
\left.\left.-\frac{1}{4} a a_{k l} a_{k l}+a_{k l}\left(2 c_{k m l m}-8 c_{m m k l}-6 c_{m l k m}\right)+\dot{b}_{k l}\right]\right) .
\end{gathered}
$$

Thus, the case of an anisotropic homogeneous shear-free cosmology is essentially defined by a Poisson-like equation (75), a Friedmann-like equation (76), a Raychaudhuri-like equation (79), and has rotation given by (78). Its variables are $\rho(t), R(t), \omega_{i j}(t), a_{i j}(t)$ and $a(t)$.

In contrast to Newtonian theory, in post-Newtonian theory the Raychaudhuri equation has the pressure entering the dynamics and so gives rise to a variety of possible solutions for $\rho(t)$ and $R(t)$. Before the only solution was that of dust. It is through the function $a(t)$, that the pressure enters into the dynamics. Without such a term the $c^{-2}$-corrections would vanish and we would be left with a system where pressure is not dynamic. There is however, a more serious reason for keeping these higher order terms and it relates to the Ellis theorem: The Raychaudhuri-like equation (79), allows for solutions with $R(t)=0$. Due to the pressure becoming dynamic, with a variation in equation of state the solutions for the density vary. Thus equations (70) and (71) no longer hold. Solutions different to dust are possible, and the rotation is no longer just a constant. Hence the Raychaudhuri equation (79) will no longer yield the result (74).

\section{Conclusion}

In this paper we have derived the post-Newtonian approximation for anisotropic homogeneous cosmologies. In contrast to the Newtonian approximation the equations are well-posed. The cosmological equations of the post-Newtonian approximation are much more in the spirit of the Bianchi types of general relativity.

We considered a particular example of the Bianchi identities - the Bianchi I cosmology. In the Newtonian theory assuming anisotropy and homogeneity leads to the well known HeckmannSchücking solutions. Howsoever, even with homogeneity, there are still not enough equations to solve for all the unknowns and some need to be supplied for all time. The post-Newtonian theory is able to overcome this problem and also allows the pressure to enter into the dynamics of the theory. Therefore the full set of possible solutions for $R(t)$ as outlined in [7], can be reproduced.

The result of pressure entering into the dynamics allows for more than just a matter dominated universe. In this way more general solutions are obtained than in the Newtonian case. In the shear-free case with rotation the Raychaudhuri equation gives rise to a singularity. In this way the Ellis theorem does not lead to contradictions, and there is hope that the solutions of the post-Newtonian approximation may all have general relativistic counterparts. In the very least we no longer have solutions to which there can be no general relativistic analogues.

It seems reasonable then to replace the Newtonian theory with the post-Newtonian approximation when considering homogeneous cosmologies, whether they be isotropic or not.

\section{References}

[1] Ellis, G. F. (1967) J. Math. Phys. 8, 1171.

[2] Heckmann, O. and Schücking, E. (1955) Zeitschrift für Astrophysik 38, 95.

[3] Heckmann, O. and Schücking, E. (1955) Handbuch der Physik LIII. Edited by S. Flügge (Springer, Berlin-Göttingen-Heidelberg), 489. 
[4] Heckmann, O. and Schücking, E. (1962) Gravitation: An Introduction to Current Research (Wiley, New York).

[5] Mac Callum, M. A. H. (1979) In An Einstein Centenary Survey: "Anisotropic and Homogeneous Relativistic Cosmologies" (ed. Hawking, S. W. and Israel, W., Cambridge University Press, Cambridge)

[6] Milne E. A. and McRae W. H., (1934) Quart. J. Math. Oxford Ser. 5 64, 73.

[7] Rainsford, T. (2000) General Relativity and Gravitation 32, 719.

[8] Raychaudhuri (1955) Phys. Rev. 98, 1123.

[9] Szekeres, P. and Rainsford, T. (2000) General Relativity and Gravitation 32389.

[10] Szekeres, P. and Rankin, R. (1977) Australian Math. Soc. B20, 114.

[11] Taub, A. (1951) Ann. Math 53, 472.

[12] Weinberg, S. (1972) Gravitation and cosmology: Principles and applications (Wiley, New York). 